\title{
An automatic linear proportional sampler based on the principles of the Theory of Sampling
}

Philippe Wavrer

CASPEO, 3 avenue Claude Guillemin, 45060 Orléans Cedex 2, France

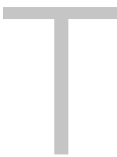

he MINEMET EAR 400 SL sampler is an automatic slurry sampler designed by Pierre Gy in the 1970s for the company Minemet. The EAR $400 \mathrm{SL}$ respects all the principles of the Theory of Sampling. This automatic in-line cross-cut sampler is particularly adapted for the sampling of slurries, such as final tailings, flotation feeds and cyclone overflows. An automatic continuous electric chain-drive system ensures a uniform cutter velocity through the material stream. The model EAR 400 presented here (made in 1973) has three fully independent influx ports; other models with four independent input ways have also been built. It is worthy of note that this particular model was displayed at the $7^{\text {th }}$ World Conference on Sampling and Blending (WCSB7), 10-12 June 2015, Bordeaux, just six months before the tragic passing of Pierre Gy in the same city.

\section{MINEMET EAR 400 SL sampler} According to the seminal publication by Pierre Gy (1981), ${ }^{1}$ sampling is said to be "proportional" when the sample, $S$, extracted from a given lot, $L$, over a flow period of duration $T_{L}$ has the following properties:

- The qualitative characteristics of the sample $S$ are "representative" of those of the lot $L$. This means the sampling process must be (at least) "correct": all particles present in the stream must have the same probability to be collected. A second demand is that sampling variance is at a specified, fit-for-purpose minimum.

The quantitative characteristics of the sample $S$ are "proportional" to those of the lot $L$ and the proportionality factor is accurately known; the sampling ratio must remain constant.

In its construction, the MINEMET EAR 400 SL sampler meets both these requirements and can therefore be considered to be a true proportional sampler. Furthermore, as three different material flows can be independently sampled, at the same time and under the same conditions, one of the major advantages of this sampler is that the sample weight ratios are an estimate of the flowrate ratios. Proportional sampling is also described in Gy's 1988 textbook. $^{2}$

\section{Description}

The EAR $400 \mathrm{SL}$ sampler consists of three main parts:

An electrically controlled mechanism for transmitting a linear reciprocat- ing uniform movement to three scoops (spoons), which are the sampling tools. This mechanism is thoroughly protected by a dust- and splash-proof hood.

- Three sampling cutters, driven simultaneously with identical velocity by the above mechanism using a fixed connecting rod.

A waterproof control box containing the electrical equipment and a timer to adjust the frequency of the sampling.

\section{Mechanism}

The uniform cutter velocity $\left(0.115 \mathrm{~m} \mathrm{~s}^{-1}\right)$ is ensured by a continuous electric chaindrive system, driven by a $0.75 \mathrm{~kW}$ electric motor associated with a speed reducer (see Figures 3 and 4). The motor is over-engineered with respect to typical loads, so as to never be slowed down even in the most irregular influx situations. Secured to the chain, a driving element engages on both sides in two slots (upper and lower) of a vertical plate (Figure 5). This one is connected to the three sampling cutters through a slide bar. The electric motor always runs in the same direction, but the slide bar (and then the sampling cutters) moves either way, depending on whether the driving element is at the top or bottom of the driving chain.

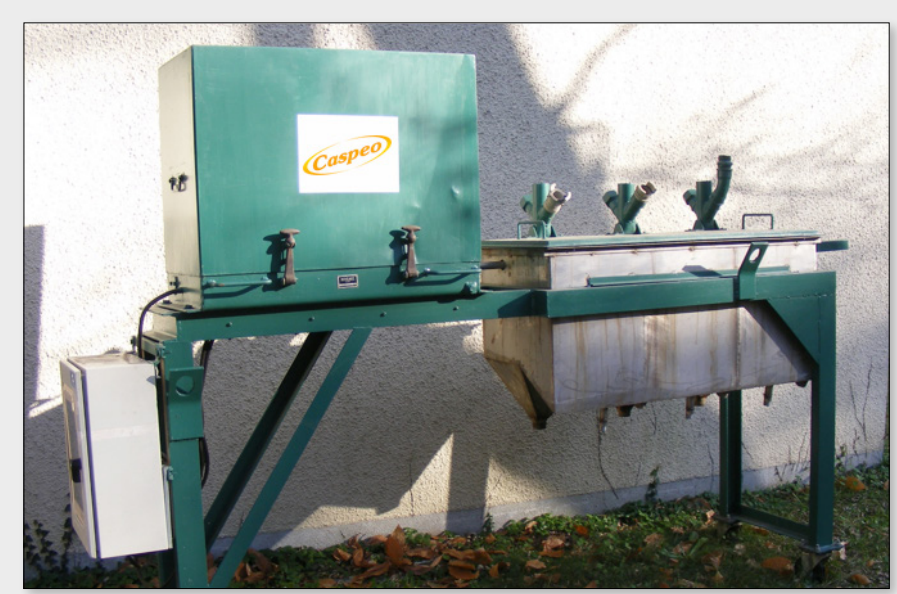

Figure 1. General view of the MINEMET EAR 400 SL slurry sampler.

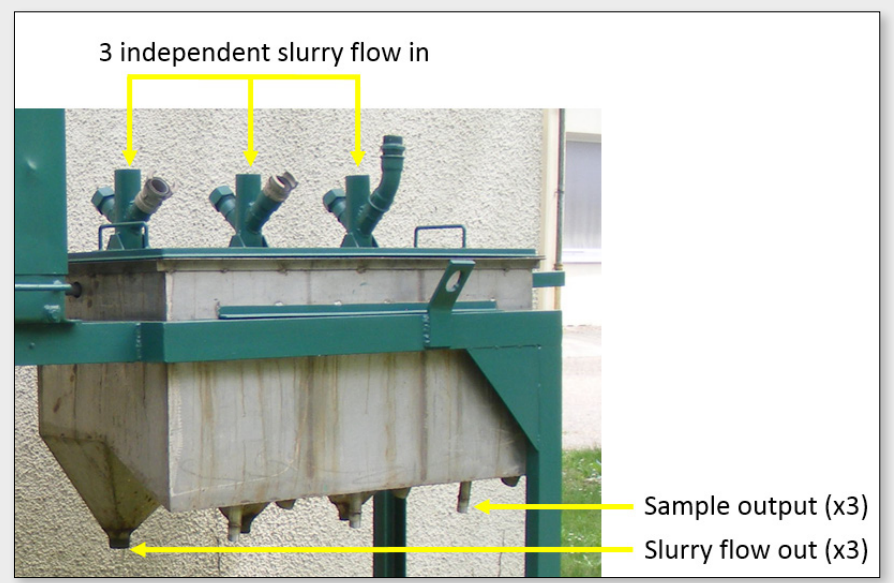

Figure 2. A linear cross-cut slurry sampler with three independent influx ports. 


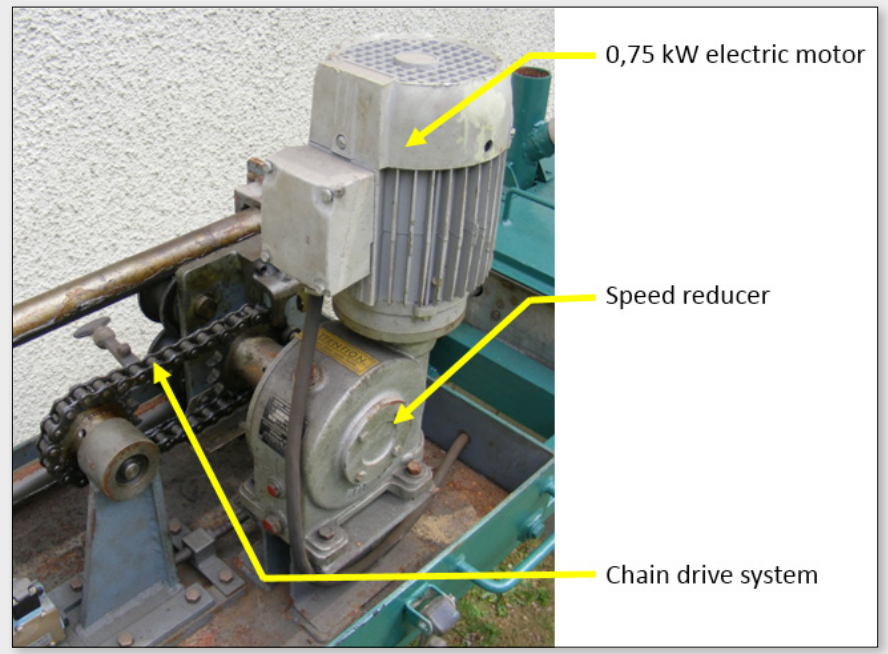

Figure 3. EAR 400 SL's automatic, continuous electric chain drive system.

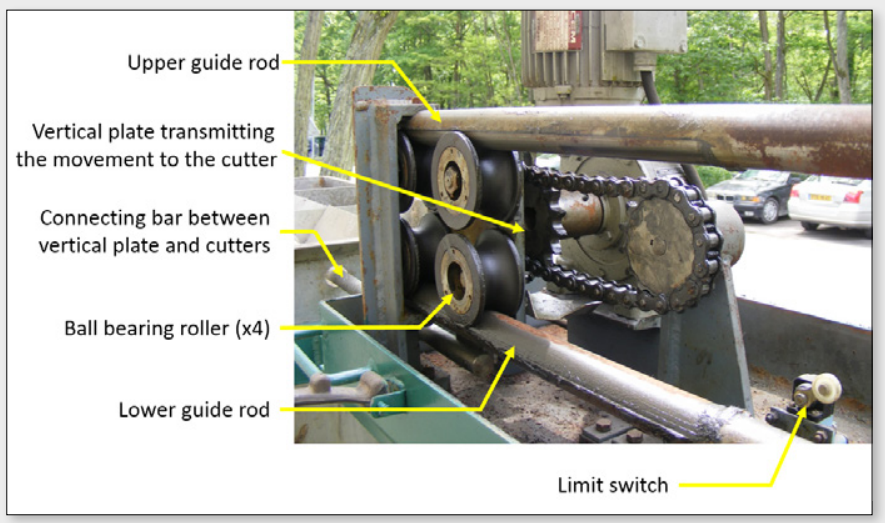

Figure 4. General view of the chain drive system. The fixed "connecting bar" transfers the drive system movement to each of the three sampling units (the most proximal one of which can be seen at the left edge of the photo)

\section{Sampling cutters}

The shape and the opening of the sampling cutter are determined based on the material to be sampled, in order to ensure sampling correctness. Concerning the present sampler, the three sampling cutters all have a $10 \times 88 \mathrm{~mm}$ rectangular opening.

Due to the length of the driving chain used, the cutters work with a stroke of $280 \mathrm{~mm}$. This magnitude ensures that the entire inflow is sampled and that the sample cutters are parked away from the material stream between each increment.

\section{Control box}

In addition to standard start/stop buttons, the control panel is equipped with:

A timer for adjusting cutter travel frequency in a range from 10 seconds to 45 hours.

A pulse counter, to express the number of increments extracted performed at any specific time, or aggregated at the conclusion of the sampling campaign.
The control box is completely waterproof and dust protected and can be locked once adjusted.

\section{Workings}

Once the sampling frequency has been set via the timer and the sampler has been powered up, a normal operating cycle is as follows:

1. Start: Movement of the cutter in one direction in order to collect one increment.

2. Motion stops at ultimate travel position (farthest parking position) for a predetermined time (counted by the timer).

3. Following the pause, movement of the cutter in the opposite direction in order to take another increment.

4. Motion stops at the closest parking position for the same predetermined pausing time.

5. Following the pause interval, commencement of the next double-cycle.

As an example, if the timer is set to two minutes, after two minutes, the timer sends an electric current pulse that starts the electric motor. The slide bar (driving the sampling three cutters) moves driven by the chain and three parallel increments are collected simultaneously. The motor is then stopped by the action of the first limit switch (see Figure 4). Two minutes later, the timer sends another electric current pulse. The electric motor starts again, always in the same direction, but the slide bar moves in the opposite direction and another increment is collected. The motor is then stopped by the action of the second limit switch. The cycle is then repeated until the STOP push button is pressed.

\section{Application example: gold flotation pilot plant}

A similar sampler to the one presented here, but with four independent influx ports, was used on a gold flotation pilot plant in French Guyana. The objective of the pilot plant project was to evaluate the possibility of recovering gold by continuous flotation

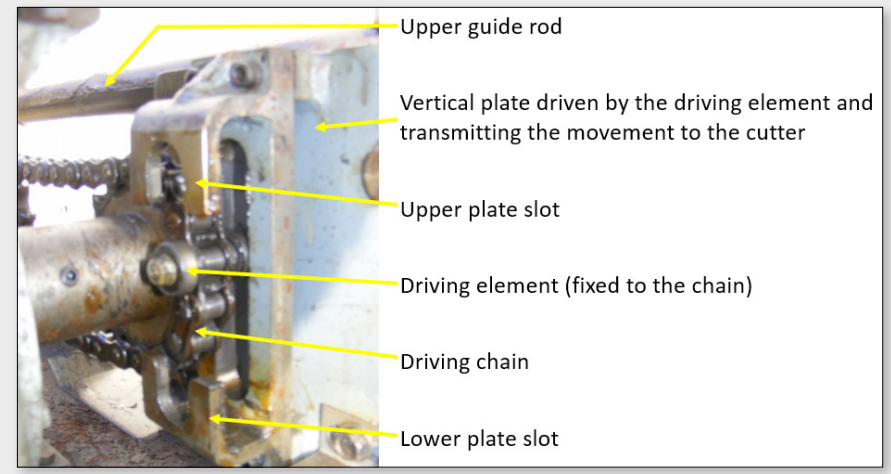

Figure 5. Detailed view of the chain-drive system.

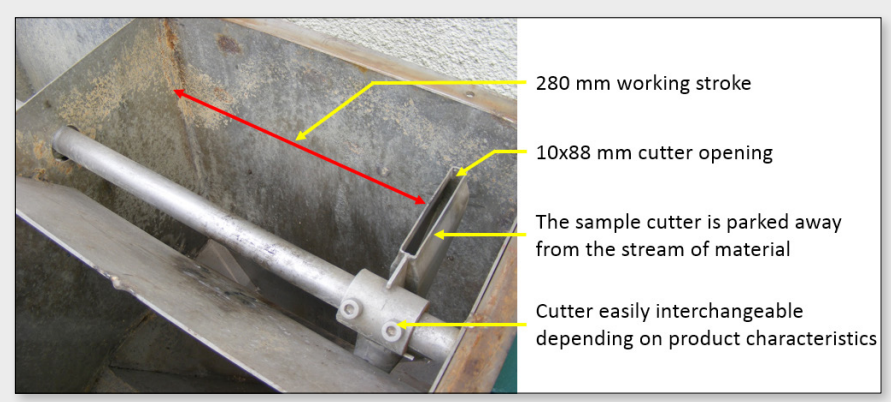

Figure 6. Sampling cutter. 


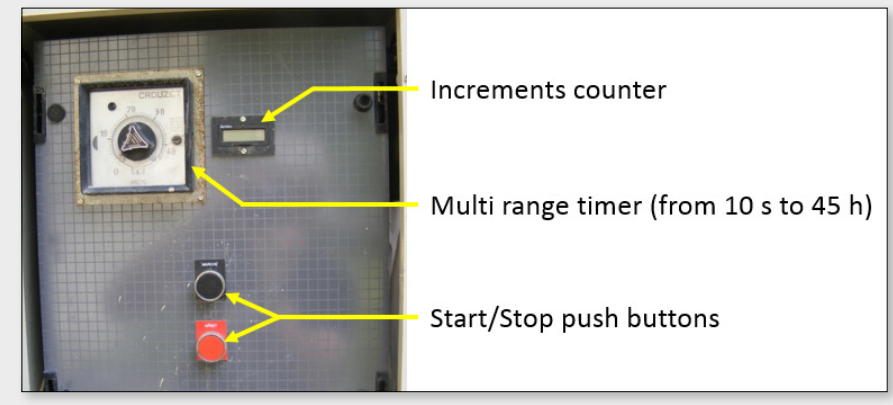

Figure 7. The 1973 control box; functionality is everything!

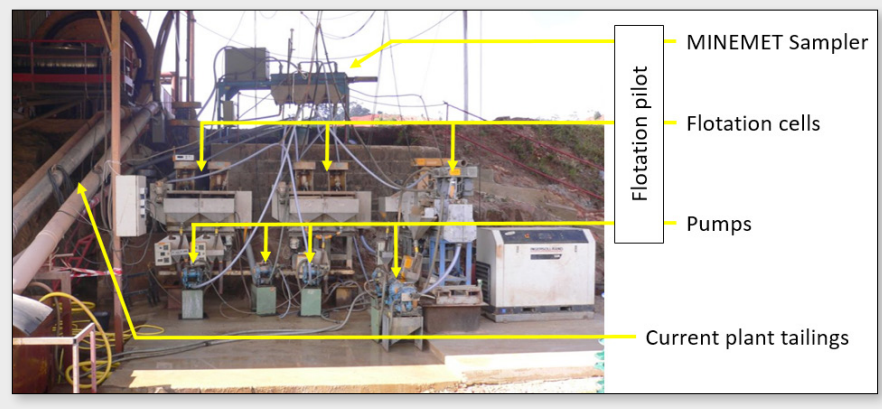

Figure 8. Overview of flotation pilot plant in French Guyana. from gravity circuit tailings. In addition to the sampler, the pilot circuit included a series of flotation cells and transfer pumps for pulp. In this case, the main advantage of the sampler is to provide sample masses whose mutual ratios are equal to the influx stream flows ratios. This property is particularly interesting with the objective of calculating material balances and gold recovery from analyses made on the collected samples. Figures 8 and 9 show an overview of the flotation pilot plant with the sampler in-line between the existing industrial plant and the pilot circuit itself and a detailed view of the automatic sampler in operation.

\section{Significance of proportional sampling}

In his theory, ${ }^{2-4}$ Gy defined "proportional" sampling as a sampling process that must be not only "correct", but also realised under conditions which allow that:

- The weight and the volume of the sample are proportional to the mass and the volume of the sampled lot,

- The proportionality factor is known with accuracy and remains constant.

With the implementation of proportional sampling, it is then possible to estimate without any bias the mass of a given particulate material lot. To achieve that, Gy has defined:

- The "time sampling ratio" $\tau^{\prime}$ of a lot $L$ :

$$
\tau^{\prime}=\frac{Q \cdot T_{i}}{T_{L}}
$$

where $T_{L}$ is the flowing time of the lot $L, Q$ is the number of increments between $t=0$ and $t=T_{L}$ and $T_{i}$ is the time of one increment.

- The "mass sampling ratio" $\tau$ :

$$
\tau=\frac{M_{E}}{M_{L}}
$$

where $M_{L}$ is the mass of the lot $L$ and $M_{E}$ is the mass of the sample $E$.

According to the theory of sampling, the sample mass $M_{E}$ being a random variable, when a sampling is correct (i.e. sampler correctly designed and built and correctly operated), it is then possible to write:

$$
m(\tau)=\tau^{\prime}
$$

where $m(\tau)$ is the mean of $\tau$.

That means that

$$
\frac{m\left(M_{E}\right)}{M_{L}}=\tau^{\prime}
$$

It can therefore be deduced that

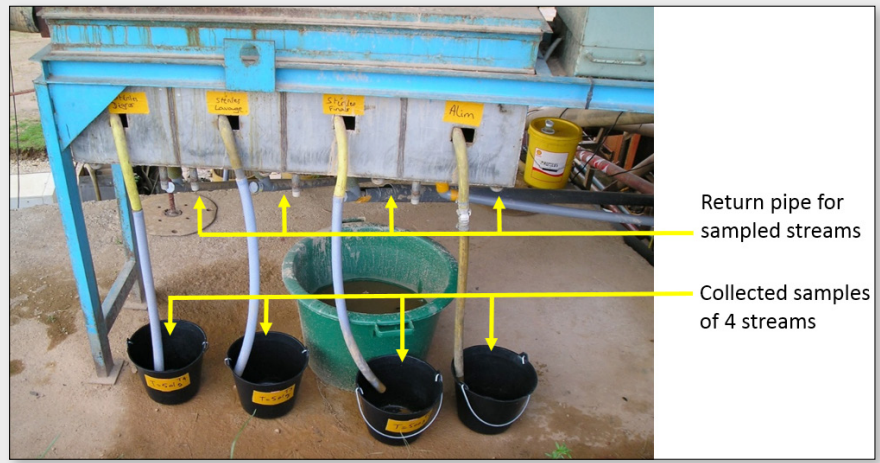

Figure 9. Simultaneous sampling of four slurry streams with mutual sampling proportionality.

$$
\frac{m\left(M_{E}\right)}{\tau^{\prime}}=M_{L}
$$

and

$$
\frac{M_{E}}{\tau^{\prime}}=M_{L}^{\prime}
$$

$M_{L}^{\prime}$ is an unbiased estimator of $M_{L}$, the mass of the lot $L$, and can be easily calculated from the mass of the sample and the time sampling ratio. $M_{E}$, the sample mass, is usually small and can be measured by weighing the sample with a precision static balance.

The time sampling ratio $\tau^{\prime}$ can be calculated with a great accuracy from sampler technical characteristics such as the cutter velocity, the length of the driving chain or the width of the cutter opening. The calculation of $\tau^{\prime}$ will be different depending on whether the sampler is running continuously or discontinuously. In this last case, the sampling frequency has to be taken into account.

Thanks to the proportional sampling, it then becomes possible to obtain in one single operation both qualitative (contents) and quantitative (masses, volumes) information and to establish very accurate and precise metallurgical balances.

\section{References}

1. P. Gy, "Proportional sampling-A new philosophy of metallurgical accounting", Int. J. Miner. Process. 8(3), 279-286 (1981).

2. P. Gy, Hétérogénéité, échantillonnage, homogénéisation. Ensemble cohérent de théories. Collection Mesures Physiques, Masson (1988).

3. P. Gy, L'échantillonnage des lots de matière en vue de leur analyse. Collection Mesures Physiques, Masson (1996).

4. P. Gy, Sampling for Analytical Purposes, 1st Edn. Wiley (1998). ISBN: 978-0-471-97956-2 